\title{
Polygenic impact of morningness on the overnight dynamics of sleep spindle amplitude
}

\section{Pesonen, Anu-Katriina}

2020-04

Pesonen, A-K, Merikanto , I , Halonen , R, Ujma , P , Makkonen , T, Räikkönen , K, Lahti , J \& Kuula , L 2020 , ' Polygenic impact of morningness on the overnight dynamics of sleep spindle amplitude ' , Genes, Brain and Behavior , vol. 19 , no. 4 , 12641 . https://doi.org/10.1111/gbb.12641

http://hdl.handle.net/10138/324748

https://doi.org/10.1111/gbb.12641

acceptedVersion

Downloaded from Helda, University of Helsinki institutional repository.

This is an electronic reprint of the original article.

This reprint may differ from the original in pagination and typographic detail.

Please cite the original version. 


\section{Polygenic impact of morningness on the overnight dynamics of sleep}

\section{spindle amplitude}

Authors: A-K Pesonen*1, I Merikanto*1,2, R Halonen ${ }^{1}$, P Ujma ${ }^{4,5}$, T Makkonen $^{6}$, K Räikkönen ${ }^{6}, \mathrm{~J} \mathrm{Lahti}^{6}, \mathrm{~L}$

Kuula ${ }^{1}$

${ }^{1}$ SleepWell Research Program, Faculty of Medicine, University of Helsinki, Helsinki, Finland

${ }^{2}$ National Institute for Health and Welfare, Helsinki, Finland

${ }^{4}$ Institute of Behavioural Sciences, Semmelweis University, Budapest, Hungary

${ }^{5}$ Epilepsy Centre, National Institute of Clinical Neurosciences, Budapest, Hungary

${ }^{6}$ Department of Psychology and Logopedics, Faculty of Medicine, University of Helsinki, Helsinki, Finland

'nared first authorship

Corresponding author: Anu-Katriina Pesonen, Faculty of Medicine, University of Helsinki, P.O. Box 21, University of Helsinki 00014, Helsinki, Finland; Tel: +358 407544942;

E-mail address: anukatriina.pesonen@helsinki.fi

This article has been accepted for publication and undergone full peer review but has not been through the copyediting, typesetting, pagination and proofreading process which may lead to differences between this version and the Version of Record. Please cite this article as doi: $10.1111 / \mathrm{gbb} .12641$ 


\section{Abstract}

Sleep spindles are thalamocortical oscillations that contribute to sleep maintenance and sleep-related brain plasticity. The current study is an explorative study of the circadian dynamics of sleep spindles in relation to a polygenic score (PGS) for circadian preference towards morningness. The participants represent the 17year follow-up of a birth cohort having both genome-wide data and an ambulatory sleep EEG measurement available $(\mathrm{N}=154$, Mean age $=16.9, \mathrm{SD}=0.1$ years, $57 \%$ girls $)$. Based on a recent genome-wide association study, we calculated a PGS for circadian preference towards morningness across the whole genome, including 354 SNPs. Stage 2 slow $(9-12.5 \mathrm{~Hz}, \mathrm{~N}=186$ 739) and fast (12.5-16 Hz, N = 135 504) sleep spindles were detected using an automated algorithm with individual time tags and amplitudes for each spindle. There was a significant interaction of PGS for morningness and timing of sleep spindles across the night. These growth curve models showed a curvilinear trajectory of spindle amplitudes: those with a higher PGS for morningness showed higher slow spindle amplitudes in frontal derivations. Overall, the findings provide new evidence on how individual sleep spindle trajectories are influenced by genetic factors associated with circadian type. The finding may lead to new hypotheses on the associations previously observed between circadian types, psychiatric problems and spindle activity. 


\section{Introduction}

Individual circadian rhythm is a biological feature governing the timing of behavioural and physiological functions, such as the sleep-wake rhythm ${ }^{1}$. Based on the variation in the timing of these functions and activity, individuals can be categorised by their circadian rhythm profile; those with earlier timed functions are considered to be more morning-oriented and the later-timed more evening-oriented ${ }^{2,3}$. Recent genomewide studies (GWAS) have identified a set of genetic loci associated with the circadian preference ${ }^{4-6}$, and provided evidence that morningness is genetically negatively correlated with both depression and schizophrenia, and positively correlated with well-being ${ }^{7}$. The same study did not find any association between these loci and sleep duration and quality measured with actigraphy, but morningness associated with earlier sleep rhythms, as in previous studies ${ }^{8}$.

Sleep EEG measurements are warranted in order to understand how genetic predispositions to circadian preference are associated not only with sleep timing, but with essential microstructures of sleep.

This information could, for example, contribute to the understanding of the associations found between between circadian types and mental health. Sleep spindles are a hallmark example of microstructure constituents: they appear as brief bursts of rhythmic thalamocortical oscillations $(9-16 \mathrm{~Hz})$ that are most prominent during stage 2 (N2) of non-rapid eye movement (NREM) sleep ${ }^{10}$. Sleep spindles have important functions, including sleep maintenance by prohibiting external stimuli inputs to the cortex ${ }^{11,12}$ and promoting long-term potentiation and enhancing sleep-related memory consolidation ${ }^{13,14}$. They also show significant heritablity ${ }^{10}$. Many psychiatric conditions, such as depression ${ }^{15}$ and schizophrenia ${ }^{16}$ have been associated with deficits in sleep spindle activity, assumed to reflect reduced brain plasticity and vulnerability for sleep disturbance. 
There are few studies showing that sleep spindles and associated sigma power in NREM sleep have an endogenous circadian rhythm. In a one-month long laboratory experiment with a forced desynchronization of the circadian rhythm, it was found that low-frequency sleep spindle amplitude reached its crest, and high-frequency sleep spindle activity reached its nadir when sleep coincided with the phase of melatonin secretion ${ }^{17}$. Similar trends were observed in two other studies, one with an experimental sleepwake paradigm, and the other studying naturally occurring sleep rhythm ${ }^{18} 19$. However, it is still unknown whether the circadian rhythm of spindles is common to all, or whether there are individual genetic tendencies that regulate sleep spindle activity over the night course. This information would be particularly important to understand individual variation in sleep regulation and associated phenomena.

The current study approached the circadian dynamics of sleep spindle activity with growth curve modelling and investigated how genetic variants associated with circadian preference are associated with both the timing and change of amplitude of sleep spindles across the night. To tackle this question, we calculated a polygenic score for morningness based on UK Biobank GWAS on circadian preference on adults ${ }^{5}$, and investigated the question in a community cohort with data from 154 adolescents. Based on our previous study on self-reported chronotype ${ }^{20}$, we hypothesized that a higher genetic tendency towards morningness would be associated with a faster decline of spindle activity towards the morning hours.

\section{Results}

\section{General sleep characteristics and spindle amplitude by sex}

Table 1 shows sleep characteristics by sex. As reported previously in the same cohort ${ }^{20}$, females had longer REM sleep duration $(P=0.02)$ and $N 2$ duration $(P=0.03)$ than males. Furthermore, fast sleep spindle amplitude was higher among females than males at the central $(P<0.001)$ and frontal $(P=0.01)$ derivation. 
There were no other differences between sexes in general sleep characteristics or in the sleep spindle amplitudes. Sex was adjusted to in all analyses.

\section{Growth curve models of sleep spindles across the night}

Frequency range 9-16 Hz: We tested the effect of time of spindle occurrence since sleep onset on spindle amplitude in linear models and non-linear (quadratic and cubic) growth curve models, with all sleep spindle frequencies pooled together, adjusting for age, sex, N2 duration, and location of derivation. Linear $\left(\mathrm{B}_{\text {linear }}=-\right.$ 0.03, Standard Error $(S E)=0.005 P=0.000001)$ and non-linear quadratic $\left(B_{\text {quadratic }}=0.01, S E=0.002, P=9.7 x\right.$ $10^{-14}$ ) growth models were statistically significant in predicting sleep spindle amplitude as a function of time since sleep onset $\left(B_{\text {cubic }}=-0.002, S E=0.002, P=0.28\right)$, the quadratic model having the best fit according to the Schwarz's Bayesian Criterion (BIC). Derivation did not moderate the association significantly ('time as quadratic since sleep onset $x$ derivation' $P=0.17$.

Frequency range 9-12.5 Hz (slow sleep spindles): Linear ( $B_{\text {linear }}=-0.03$, Standard Error $(\mathrm{SE})=0.01 \mathrm{P}=3.1 \times 10^{-}$ $\left.{ }^{8}\right)$ and non-linear quadratic $\left(B_{\text {quadratic }}=0.01, \mathrm{SE}=0.002, \mathrm{P}=3.4 \times 10^{-7}\right)$ growth models were statistically significant in predicting sleep spindle amplitude as a function of time since sleep onset $\left(B_{\text {cubic }}=0.008, S E=\right.$ $0.002, P=0.67)$, again quadratic model having the best fit $(\mathrm{BIC})$. Derivation did not moderate the association significantly ('time as quadratic since sleep onset $x$ derivation' $P=0.70$ ).

Frequency range $12.5-16 \mathrm{~Hz}$ (fast sleep spindles): Linear $\left(\mathrm{B}_{\text {linear }}=-0.02\right.$, Standard Error $(\mathrm{SE})=0.001, \mathrm{P}=0.013$ ) and non-linear quadratic $\left(B_{\text {quadratic }}=0.01, S E=0.003, P=1.8 \times 10^{-7}\right)$ and cubic growth model $\left(B_{\text {cubic }}=-0.007, S E\right.$ $=0.002, \mathrm{P}=0.002$ ) were statistically significant in predicting sleep spindle amplitude as a function of time since sleep onset. The best fit according to the Schwarz's Bayesian Criterion (BIC) was attained with the quadratic model. Derivation moderated the association significantly ('time as quadratic since sleep onset $\mathrm{x}$ derivation' $P=0.02$ ). Post hoc tests indicated a significant quadratic model in central $\left(B_{\text {quadratic }}=0.12, S E 0.02\right.$, 
$\left.P=1.9 \times 10^{-8}\right)$ and in frontal $\left(B_{\text {quadratic }}=0.06, S E 0.03, P=0.03\right)$ derivations. Figure 1 shows the trajectories of sleep spindles in frontal (Panel A) and central (Panel B) derivations.

PGS for morningness and the average spindle amplitude overnight in the frequency range 9-16 $\mathrm{Hz}$

As Table 2 shows, higher PGS for morningness was not associated with the average sleep spindle amplitude across the night $P=0.93$ ) in the linear mixed model, with all sleep spindle frequencies were pooled together, adjusting for age, sex, stage 2 duration, and derivation. The analysis for the association between PGS for morningness and fast sleep spindle amplitude was statistically significant $(P=0.038 ; P=0.26$ for slow sleep spindle amplitude). Thus, higher PGS for morningness was associated with lower average fast sleep spindle amplitude across the night, although the association did not survive the Bonferroni correction (Table 2).

\section{PGS for morningness and timing of spindle amplitude in the frequency range 9-16 $\mathrm{Hz}$}

Regarding spindles in the entire frequency range $(9-16 \mathrm{~Hz})$, the interaction between 'PGS for morningness and time since sleep onset as quadratic' was significantly associated with sleep spindle amplitude $(\mathrm{P}=$ 0.00002; Table 2). Figure 2 displays the interaction with the PGS score divided in three categories, based on \pm 1 standard deviations groups. It shows the highest amplitudes at the sleep onset for all PGS groups, and a steeper decline among those with high PGS for morningness of sleep spindle amplitude in the last decile of the sleep time. The three-way interaction with derivation ('PGS for morningness $x$ derivation $\times$ time from sleep onset as quadratic', was not significant $(P=0.57$; Table 2$)$.

\section{PGS for morningness and timing of spindle amplitude in the frequency range 9-12.5 Hz}

Regarding spindles in the slow frequency range $(9-12.5 \mathrm{~Hz})$, the interaction between 'PGS for morningness $x$ time from sleep onset as quadratic' was significantly associated with slow sleep spindle amplitude $(P=$ 0.000005; Table 2). The three-way interaction with derivation ('PGS for morningness $x$ derivation $\times$ time from 
sleep onset as quadratic', was significant $(P=0.004)$, and the post-hoc interaction ('PGS for morningness $x$ time from sleep onset as quadratic') was significant in the central derivation $(B=-0.02, S E=0.005, P=$ $0.000002 ; \mathrm{P}=0.07$ for the frontal derivation). Figure 3 displays the interaction with the PGS score divided in three categories, based on \pm 1 standard deviations groups. Figure $3 \mathrm{~A}$ (frontal slow) shows significantly higher spindle amplitude among those with high PGS for morningness both at sleep onset, and in six deciles of time thereafter. Figure 3B (central slow) shows a more mixed picture, with the lowest amplitudes for the group with high PGS for morningness both at sleep onset and in the last decile.

\section{PGS for morningness and timing of spindle amplitude in the frequency range 12.5-16 $\mathrm{Hz}$}

As Table 2 shows, for spindles in the fast range $(12.5-16 \mathrm{~Hz})$, the interaction between 'PGS for morningness and time since sleep onset as quadratic' was not associated with sleep spindle amplitude $(P=0.42)$. The EEG measurement derivation did not moderate the effect on fast spindle amplitude ('derivation $\times$ PGS for morningness $x$ time from sleep onset as quadratic', $P=0.41$.

\section{Materials and Methods}

\section{Participants}

The study sample is derived from a Finnish community-based cohort of 1049 healthy singletons born Detween March and November 1998 in Helsinki, Finland. The details of the cohort are described in more detail in previous reports ${ }^{21-24}$. We invited those cohort members who participated in the previous follow-up at age 12 in years 2014-15 and lived within the $30 \mathrm{~km}$ radius from Helsinki ( $N=279,77.1 \%$ of the participants of the 12-year follow-up). Of them, 197 (70.6 \%) participated at the age of 17 (mean age $=16.9$, SD $=0.1$ years). All participants were Caucasian. 
The analytic sample comprised 154 ( 87 females and 67 males) adolescents who had both complete records of an overnight sleep EEG measurement at the age of 17 and genetic samples extracted at the age of 12. The Ethics Committee for Children and Adolescents' Diseases and Psychiatry at the Helsinki University Central Hospital approved the study protocol. All participants and their parents gave their written informed consent.

\section{Sleep EEG recording}

Overnight polysomnographic sleep recordings (PSG) were conducted in the homes of the participants with SOMNOscreen plus (SOMNOmedics $\mathrm{GmbH}$, Germany). The participants were instructed to follow their own sleep schedule. Electroencephalography (EEG) was recorded with gold cup electrodes at 6 EEG locations (frontal (F) hemispheres: F3, F4; central (C) hemispheres: C3, C4; occipital (O) hemispheres: O1, O2) and two channels for the mastoids ( $\mathrm{A} 1, \mathrm{~A} 2$ ) according to the standardized 10/20 system. The electro-oculogram (EOG) and the electromyogram (EMG) were measured by using disposable adhesive electrodes (Ambu Neuroline 715, Ambu A/S, Denmark), two locations for EOG and three locations for EMG. In addition, an online reference $\mathrm{Cz}$ and a ground electrode in the middle of the forehead were used. The sampling rate was $256 \mathrm{~Hz}$ (the hardware filter bandwidth for SOMNOscreen plus is $0.2-35 \mathrm{~Hz}$ ). PSG data was scored manually using the DOMINO program (v2.7; SOMNOmedics GmbH, Germany) in 30-sec epochs into N1, N2, N3 and REM according to AASM guidelines (The AASM Manual for the Scoring of Sleep and Associated Events).

\section{Spindle Detection and Power Spectral Density Analysis}

The manually scored PSG signals were converted to EDF format in DOMINO software and then further analysed by using functions of EEGlab 14.1.2 b $^{25}$ running on Matlab R2018a (The Mathworks Inc., USA). All signals were digitally offline filtered with pass band of $0.5-35 \mathrm{~Hz}$ (Hamming windowed sinc zero-phase FIR filter, cut-off (-6dB) $0.25 \mathrm{~Hz}$ and $39.3 \mathrm{~Hz}$ respectively) and re-referenced to the average signal of A1 and A2 
electrodes. Electrodes located at F3, F4, C3, C4 were included in the analysis. Only epochs with impedance equal to or lower than $10 \mathrm{k} \Omega$ on all scalp electrodes and both mastoids were included in the analyses.

Spindles were computationally extracted with a method based on an automated detection algorithm described by Ferrarelli ${ }^{26}$. The spindle analysis was conducted in valid N2 epochs in two different frequency bands (slow: $9-12.5 \mathrm{~Hz}$, and fast: $12.5-16 \mathrm{~Hz}$ ) in order to differentiate between slow and fast spindles. Before applying an amplitude threshold for spindles, the pre-processed EEG data were further filtered using the above-mentioned frequency bands separately. The threshold values for detecting spindles in each channel were defined by the mean of the channel amplitude $(\mu \mathrm{V})$ multiplied with 6 (higher) including all valid epochs ( $\mathrm{N} 2$ and mastoid impedance $\leq 10 \mathrm{k} \Omega$ ). The spindle's amplitude was required to stay over the mean channel amplitude multiplied by 2 (lower) for $250-1000 \mathrm{~ms}$ (duration limit) in both directions from the peak maximum. Thus, we used channel-wise threshold definitions, taking into account that signal amplitude may vary across the channels. Before and after each detected spindle, signal amplitude was required to stay under the lower threshold for $78.1 \mathrm{~ms}$ which is approximately the duration of one period of sine at $13 \mathrm{~Hz}$. This was done in order to prevent false alarms in spindle detection. Sleep spindle amplitude was measured separately for central and frontal derivations. In order to obviate interindividual amplitude differences from the overnight dynamics of spindle amplitudes, standardized amplitude scores for individual spindles were culated separately for each subject. Derivation-specific spindle mean amplitudes were calculated only if at least 10 minutes of N2 EEG data was available. Spindles with amplitude exceeding $80 \mu \mathrm{V}$ or 6 SD from the individual mean were excluded ( $1.01 \%$ of spindles). Altogether, our data comprised 321243 spindles ( $\mathrm{N}_{\text {slow }}$ $=186739$ and $\mathrm{N}_{\text {fast }}=135$ 504. Compared to our previous analysis of spindle distribution across the night ${ }^{27}$, the current spindle detection method is less strict in terms of the spindle threshold value ( 6 vs. 8 times channel amplitude), and the contrary to our previous study, the amplitudes were standardized within the 
subject's values across the night. Similarly to existing knowledge ${ }^{18}$, we found a lower average spindle frequency in frontal vs. central derivations $(11.7 \mathrm{~Hz}$ vs. $12.6 \mathrm{~Hz}$ respectively, $\mathrm{P}<0.001)$.

\section{Genotyping, SNP selection and calculation of the weighted polygenic score}

DNA was extracted from blood (22\%) and saliva samples (78\%) collected at the $2009-2011$ follow-up and the genotyping was performed with the Illumina OmniExpress Exome 1.2 bead chip at the Tartu University, Estonia, in September 2014, according to the standard protocols. Genomic coverage was extended by imputation using the 1000 Genomes Phase I integrated variant set (v3 / April 2012; NCBI build 37 / hg19) as the reference sample and IMPUTE2 software. Before imputing the following quality control filters were applied: SNP clustering probability for each genotype $>95 \%$, Call rate $>95 \%$ individuals and markers (99\% for markers with minor allele frequency (MAF) < $5 \%$ ), MAF > $1 \%$, Hardy-Weinberg equilibrium (HWE) $P>1 \times 10-6$. Moreover, heterozygosity, sex check and relatedness checks were performed and any discrepancies removed $(N=2)$. We calculated the polygenic score $(P G S)$ for morningness based on a recent GWAS $^{5}$ across whole genome. We computed the PGS for morningness using the beta and P-values from summary statistics of this GWAS for self-reported chronotype, based on data from 128,266 British adults, aged 37 to 73 years, from the UK Biobank study and replicated in 89,283 23andMe participants ${ }^{5}$. Before calculating the PGS, clumping was performed with plink 2.0 (http://www.cog-genomics.org/plink/2.0/) in order to remove the SNPs which are in linkage disequilibrium with each other ( $r^{2}$ value 0.1 used according to the default value in PRSice software). For the PGS analysis, we used the statistical analysis software package PRSice v2.2.0. Best fit PGS for genetic tendency towards morningness was gained with a $p$-value threshold of 0.0001 including 354 SNPs. The correlation of this PGS with the self-reported chronotype was modest in our sample $(r=0.157 p=0.025)$. 


\section{Statistical analysis}

We analysed the sex differences in general sleep characteristics and sleep spindle amplitude with one-way ANOVA. We used unconditional growth curve analyses ${ }^{28}$ to study whether the sleep spindle amplitude was associated with time distribution of spindles during the night. The time was calculated as spindle occurrence time as seconds from the individual sleep onset time. We modelled the time both as linear, and as in higherorder quadratic (time $\times$ time) and cubic (time $\times$ time $\times$ time) terms. If the time effect was significant in predicting the sleep spindle amplitude, we analysed the interaction between PGS for morningness and time, and between PGS for morningness, time and derivation in predicting spindle characteristics. We used intercept and time from sleep onset as random-effect variables. This indicates that the slopes were defined individually taking into account the effect of sleep duration. We used Bonferroni corrections to adjust for multiple testing in the analyses concerning the associations between PGS and sleep spindle amplitude.

\section{Discussion}

Our explorative study used growth curve modelling to explore how sleep spindle activity is organized across the night in relation to polygenic score for morningness, and taking into account both the individual sleep time and the individual's own level of spindle activity. While there were no large differences in the average level of sleep spindle amplitudes, we found associations between the genetic predisposition for circadian preference and temporal sleep spindle dynamics across the night. Specifically, individuals with a higher genetic propensity for morningness displayed higher spindle amplitudes in the low frequency range for the most part of the night in the frontal derivation. The findings did not consistently support our hypothesis of a steeper decline in spindle amplitudes towards the morning in individuals with a higher genetic propensity towards morningness, although a trend was observed specifically in central low frequency spindles. Together, 
these findings show how individual genetic tendency towards morningness/eveningness extends its influence from sleep timing to sleep microstructure events.

Our finding of the overnight dynamics of sleep spindle amplitude being influenced by PGS for circadian preference expands prior knowledge of sleep spindle amplitude dynamics across the night and circadian periods ${ }^{17-19}$. While these previous studies have examined universal characteristics of sleep spindle function, the current study contributes to the field by showing how individual genetic contributions related to circadian preference modify the sleep spindle trajectories. Previous experimental studies ${ }^{17,18}$ both showed how the amplitude of sleep spindles is modulated by the endogenous circadian period, the amplitude of spindles peaking the phase of melatonin secretion for slow spindles, and being at its nadir for fast spindles. This trend was echoed in the current study such that there was a clear decreasing slope of specifically slow spindles from the sleep onset.

There are some studies that have focused on self-reported circadian preference and sleep dynamics across the night. For example, it has been reported that morning-types express elevated homeostatic responsivity to sleep pressure changes, which is reflected in higher initial level and faster dissipation of slow-wave activity ${ }^{29-32}$ and larger decrease in sigma power ${ }^{30}$. As sleep spindles are regarded to have a key role in sleep maintenance by prohibiting external stimuli inputs to the cortex ${ }^{11,12}$, a steeper decrease of amplitude towards the end of sleep time in genetic morning-types would indicate earlier homeostatic readiness for awakening. However, the current study did not strongly support this hypothesis, although a trend for steeper decline was observed in slow central spindle amplitudes in the group of genetic morning types. Indeed, our previous study ${ }^{20}$ based on a self-reported circadian preference showed a more distinct association between self-reported morningness and steeper decline of spindles towards the morning ${ }^{27}$ than the current study. However, in the current study, in the light of new data ${ }^{33}$ we refined the 
cut-off for fast spindles from 13 to $12.5 \mathrm{~Hz}$, and refined the spindle detection method, thus our two studies are not entirely paralleled in terms of sleep spindle definition. Of the previous studies examining the course of spindle-related sigma power in different circadian types, Mongrain et al. ${ }^{29}$ also reported a curvilinear association with time across the night. They found a steeper increase of low sigma power $(12-14 \mathrm{~Hz})$ during the first three NREM cycles, and a steeper decrease during the fourth NREM cycle among morning-versus evening-types. The circadian types differed specifically in parietal but not in frontal low sigma power profiles. Comparisons between studies are not straightforward though: the low and high sigma frequency ranges used in their study ${ }^{29}$ differed from our definition of slow and fast spindles, their study had less participants, and their sample consisted of older individuals aged 19 to 34 years. The age effect is important to acknowledge when comparing the studies, as a recent study on 11630 individuals showed that spindle frequency is profoundly affected by age ${ }^{10,34}$. Also, spindles of low $(\sim 11 \mathrm{~Hz})$ and fast $(\sim 15 \mathrm{~Hz})$ frequencies show independent topography as well as differing overnight dynamics ${ }^{10}$.

Another interesting perspective related to the association observed between higher slow spindle activity along with a higher PGS for morningness, is related to the well-documented association between morningness and a lower risk for mental health problems. Together with the reports showing deficits in spindle activity in psychiatric conditions, such as depression ${ }^{15}$ and schizophrenia ${ }^{16}$, the current dings open up new hypotheses of their common genetic pathways. In our recent study in the same adolescent cohort as reported here, we showed that fast sleep spindle amplitude and density were associated with a PGS for schizophrenia, and slow sleep spindle duration and amplitude with the CACNA1I gene variants ${ }^{35}$. The CACNA1l gene encodes the T-type calcium channels in thalamic reticular nucleus generating sleep spindles ${ }^{36}$. This and other genes influencing for example the regulation of thalamocortical networks could present future gene candidates for examining the association between circadian rhythms and sleep spindle characteristics. 
The overall picture of particular genetic variants influencing sleep microstructure, such as sleep spindles, is still largely unknown. Further investigations are needed to understand the complex interactions of homeostatic pressure, circadian pacemakers and genetic influence in the association with sleep spindles and circadian rhythms. Twin studies have shown sleep EEG characteristics to be highly heritable traits, with one study suggesting up to a $96 \%$ heritability estimate at the $8-16 \mathrm{~Hz}$ power spectra range $^{37}$. One recent twin study found the genetic influence to be stronger regarding slow rather than fast sleep spindles ${ }^{38}$. While the mechanisms for this are unclear, our overall findings echoed the notion that genetic mechanisms play a part in spindle activity.

\section{Strengths and limitations}

Our sample provides a unique platform to study the question of genetic regulation of sleep spindles, as only few cohorts have both sleep EEG and genome-wide data available. As a further strength, the participants slept according to their normal schedule in their own home environment, which increases the validity of the studied sleep parameters. We also had a very good control of the EEG data quality, and excluded any epochs with poor impedance from the spindle analyses. The sample has a homogeneous age distribution, which increases the specificity of our analyses, as age can be a major confounding factor regarding research on spindle activity ${ }^{34}$. However, this may also prevent generalizability of the current findings to other age groups. Regarding the age-specificity of chronotype, we have previously reported that the PGS for morningness was associated the continuity of sleep timing from age 8 to 17 years, thus from childhood to late adolescence ${ }^{8}$.

As a limitation, our study only includes sleep EEG data for one night. Recordings covering two or several nights would provide a better representation of the night-to-night stability of sleep spindles. Second, while we have been able to investigate such a large and healthy sample in optimal conditions, there may still be further confounding factors which influence the participants' spindle activity. Furthermore, our 
study is only correlative in its nature and does not imply any potential mechanism for the findings: the genetic variation relating to circadian preference is likely to influence sleep behaviour, which in turn mediates spindle activity. Thus, the associations we report may be a representation of these pathways rather than direct causalities. Finally, it is not clear whether spindles manifesting in different derivations originate from functionally different thalamic sources, or whether they are reflections of the same origin.

\section{Conclusions}

Our results indicate that polymorphisms in different genetic variants influencing circadian preference ${ }^{5}$ are also associated with sleep spindle activity. The main finding revealed that those with a stronger genetic tendency towards morningness displayed higher spindle amplitudes in the low frequency range for the most part of the night in the frontal derivation. These findings contribute to the understanding of individual, genetic-driven differences in the circadian regulation of sleep EEG oscillations. As sleep spindles contribute to sleep maintenance and brain plasticity, the found associations may underlie both associations found between low spindle activity and mental health problems.

\section{Conflict of Interests statement}

This study was conducted at the University of Helsinki and was funded by The Academy of Finland and the Finnish Cultural Foundation. The funders had no role in study design, data collection and interpretation, or the decision to submit the work for publication. There are no competing financial interests. All authors have seen and approved the manuscript. There are no conflicts of interest.

\section{References}


1. Roenneberg T, Kuehnle T, Juda M, et al. Epidemiology of the human circadian clock. Sleep Med Rev. 2007; 11,429-38.

2. Horne JA, Ostberg $O$. A self assessment questionnaire to determine Morningness Eveningness in human circadian rhythms. International Journal of Chronobiology. 1976; 4,97-110.

3. Roenneberg T, Wirz-Justice A, Merrow M. Life between clocks: daily temporal patterns of human chronotypes. J Biol Rhythms. 2003; 18,80-90.

4. Hu Y, Shmygelska A, Tran D, Eriksson N, Tung JY, Hinds DA. GWAS of 89,283 individuals identifies genetic variants associated with self-reporting of being a morning person. Nat Commun. 2016; 7,10448 .

5. Jones SE, Tyrrell J, Wood AR, et al. Genome-Wide Association Analyses in 128,266 Individuals Identifies New Morningness and Sleep Duration Loci. PLoS Genet. 2016; 12,e1006125.

6. Lane JM, Vlasac I, Anderson SG, et al. Genome-wide association analysis identifies novel loci for chronotype in 100,420 individuals from the UK Biobank. Nat Commun. 2016; 7,10889.

7. Jones SE, Lane JM, Wood AR, et al. Genome-wide association analyses of chronotype in 697,828 individuals provides insights into circadian rhythms. Nat Commun. 2019; 10,343.

8. Merikanto I, Lahti J, Kuula L, et al. Circadian preference and sleep timing from childhood to adolescence in relation to genetic variants from a genome-wide association study. Sleep Med. 2018; 50,3641.

9. Dijk DJ, Czeisler CA. Contribution of the circadian pacemaker and the sleep homeostat to sleep propensity, sleep structure, electroencephalographic slow waves, and sleep spindle activity in humans. J Neurosci. 1995; 15,3526-38.

10. Purcell SM, Manoach DS, Demanuele C, et al. Characterizing sleep spindles in 11,630 individuals from the National Sleep Research Resource. Nat Commun. 2017; 8,15930.

11. Dang-Vu TT, Bonjean M, Schabus M, et al. Interplay between spontaneous and induced brain activity during human non-rapid eye movement sleep. Proc Natl Acad Sci U S A. 2011; 108,15438-43.

12. Cote KA, Epps TM, Campbell KB. The role of the spindle in human information processing of high-intensity stimuli during sleep. J Sleep Res. 2000; 9,19-26.

13. Niethard N, Burgalossi A, Born J. Plasticity during Sleep Is Linked to Specific Regulation of Cortical Circuit Activity. Front Neural Circuits. 2017; 11,65.

14. Lindemann C, Ahlbeck J, Bitzenhofer SH, Hanganu-Opatz IL. Spindle Activity Orchestrates Plasticity during Development and Sleep. Neural Plast. 2016; 2016,5787423.

15. Hamann C, Rusterholz T, Studer M, Kaess M, Tarokh L. Association between depressive svmptoms and sleep neurophysiology in early adolescence. J Child Psychol Psychiatry. 2019.

16. Baran B, Karahanoglu FI, Mylonas D, et al. Increased Thalamocortical Connectivity in Schizophrenia Correlates With Sleep Spindle Deficits: Evidence for a Common Pathophysiology. Biol Psychiatry Cogn Neurosci Neuroimaging. 2019; 4,706-14.

17. Dijk DJ, Shanahan TL, Duffy JF, Ronda JM, Czeisler CA. Variation of electroencephalographic activity during non-rapid eye movement and rapid eye movement sleep with phase of circadian melatonin rhythm in humans. J Physiol. 1997; 505 ( Pt 3),851-8.

18. Knoblauch V, Martens W, Wirz-Justice A, Krauchi K, Cajochen C. Regional differences in the circadian modulation of human sleep spindle characteristics. Eur J Neurosci. 2003; 18,155-63.

19. Bodizs R, Kormendi J, Rigo P, Lazar AS. The individual adjustment method of sleep spindle analysis: methodological improvements and roots in the fingerprint paradigm. J Neurosci Methods. 2009; 178,205-13. 
20.

Merikanto I, Kuula L, Makkonen T, et al. Circadian preference towards morningness is associated with lower slow sleep spindle amplitude and intensity in adolescents. Scientific reports. 2017; 7,14619 .

21. Kuula L, Pesonen AK, Kajantie E, et al. Sleep and Lipid Profile During Transition from Childhood to Adolescence. J Pediatr. 2016.

22. Merikanto I, Pesonen AK, Kuula L, et al. Eveningness as a risk for behavioral problems in late adolescence. Chronobiol Int. 2017; 34,225-34.

23. Pesonen AK, Martikainen S, Heinonen K, et al. Continuity and Change in Poor Sleep from Childhood to Early Adolescence. Sleep. 2014; 37,289-97.

24. Raikkonen K, Matthews KA, Pesonen AK, et al. Poor sleep and altered hypothalamicpituitary-adrenocortical and sympatho-adrenal-medullary system activity in children. The Journal of clinical endocrinology and metabolism. 2010; 95,2254-61.

25. Delorme A, Makeig S. EEGLAB: an open source toolbox for analysis of single-trial EEG dynamics including independent component analysis. J Neurosci Methods. 2004; 134,9-21.

26. Ferrarelli F, Peterson MJ, Sarasso S, et al. Thalamic dysfunction in schizophrenia suggested by whole-night deficits in slow and fast spindles. Am J Psychiatry. 2010; 167,1339-48.

27. Merikanto I, Kuula L, Makkonen T, et al. Circadian preference towards morningness is associated with lower slow sleep spindle amplitude and intensity in adolescents. Sci Rep. 2017; 7,14619.

28. Panik M. Growth Curve Modeling: Theory and Applications2013.

29. Mongrain V, Carrier J, Dumont M. Difference in sleep regulation between morning and evening circadian types as indexed by antero-posterior analyses of the sleep EEG. Eur J Neurosci. 2006; 23,497-504.

30. Mongrain V, Dumont M. Increased homeostatic response to behavioral sleep fragmentation in morning types compared to evening types. Sleep. 2007; 30,773-80.

31. Kerkhof GA. Differences between morning-types and evening-types in the dynamics of EEG slow wave activity during night sleep. Electroencephalogr Clin Neurophysiol. 1991; 78,197-202.

32. Mongrain V, Carrier J, Dumont M. Chronotype and sex effects on sleep architecture and quantitative sleep EEG in healthy young adults. Sleep. 2005; 28,819-27.

33. Pesonen A-K, Ujma P, Halonen R, Räikkönen K, Kuula L. The associations between spindle characteristics and cognitive ability in a large adolescent birth cohort. Intelligence. 2019; 72,13-9.

34. Clawson BC, Durkin J, Aton SJ. Form and Function of Sleep Spindles across the Lifespan. Neural Plast. 2016; 2016,6936381.

35. Merikanto I, Utge S, Lahti J, et al. Genetic risk factors for schizophrenia associate with sleep spindle activity in healthy adolescents. J Sleep Res. 2018,e12762.

36. Astori $\mathrm{S}$, Wimmer RD, Prosser HM, et al. The $\mathrm{Ca}(\mathrm{V}) 3.3$ calcium channel is the major sleep spindle pacemaker in thalamus. Proc Natl Acad Sci U S A. 2011; 108,13823-8.

37. De Gennaro L, Marzano C, Fratello F, et al. The electroencephalographic fingerprint of sleep is genetically determined: a twin study. Ann Neurol. 2008; 64,455-60.

38. Adamczyk M, Genzel L, Dresler M, Steiger A, Friess E. Automatic Sleep Spindle Detection and Genetic Influence Estimation Using Continuous Wavelet Transform. Front Hum Neurosci. 2015; 9,624. 
Table 1. Polysomnography-based sleep characteristics and sleep spindle amplitude by sex analyzed with one-way ANOVA. SD refers to standard deviation.

\begin{tabular}{|c|c|c|c|}
\hline & \multirow{2}{*}{$\begin{array}{l}\text { Girls }(\mathrm{N}=87) \\
\text { Mean } \pm \mathrm{SD} / \%\end{array}$} & \multicolumn{2}{|l|}{ Boys $(\mathrm{N}=67)$} \\
\hline & & Mean \pm SD $/ \%$ & $P$ \\
\hline Age & $16.9 \pm 0.1$ & $16.9 \pm 0.1$ & 0.7 \\
\hline Sleep duration (hh:mm) & $7: 48 \pm 1: 06$ & $7: 30 \pm 1: 04$ & 0.08 \\
\hline Wake after sleep onset (WASO) & $0: 31 \pm 0: 23$ & $0: 34 \pm 0: 26$ & 0.5 \\
\hline REM duration (hh:mm) & $1: 42 \pm 0: 29$ & $1: 30 \pm 0: 30$ & 0.02 \\
\hline NON-REM duration (hh:mm) & $6: 06 \pm 0: 48$ & $5: 59 \pm 0: 52$ & 0.4 \\
\hline Stage 1 duration (hh:mm) & $0: 48 \pm 0: 22$ & $0: 54 \pm 0: 22$ & 0.06 \\
\hline Stage 2 duration (hh:mm) & $3: 16 \pm 0: 39$ & $3: 02 \pm 0: 42$ & 0.03 \\
\hline Stage 3 duration (hh:mm) & $2: 03 \pm 0: 28$ & $2: 03 \pm 0: 27$ & 0.9 \\
\hline Central slow amplitude $(\mu \mathrm{V})$ & $20.4 \pm 4.3$ & $20.1 \pm 4.1$ & 0.7 \\
\hline Frontal slow amplitude $(\mu \mathrm{V})$ & $19.2 \pm 4.6$ & $19.3 \pm 4.0$ & 0.9 \\
\hline Central fast amplitude $(\mu \mathrm{V})$ & $18.3 \pm 4.6$ & $14.6 \pm 4.0$ & $8.5 \times 10^{-7}$ \\
\hline Frontal fast amplitude $(\mu \mathrm{V})$ & $12.1 \pm 3.2$ & $11.0 \pm 2.2$ & 0.01 \\
\hline
\end{tabular}


Table 2. Associations between PGS and sleep spindle amplitude

\begin{tabular}{cllll}
\hline & Main effect & & 'PGS x time as quadratic' & $\begin{array}{l}\text { 'PGS x time as quadratic } \\
\text { x derivation' }\end{array}$ \\
\hline & $\mathrm{B}(\mathrm{SE})$ & P-value & P-value & P-value \\
\hline Frequency range & & & & \\
$9-16 \mathrm{~Hz}$ & $0.001(0.01)$ & 0.88 & $0.00002^{*}$ & 0.57 \\
$9-12.5 \mathrm{~Hz}$ & $0.01(0.01)$ & 0.26 & $0.000005^{* * *}$ & $0.004^{*}$ \\
$12.5-16 \mathrm{~Hz}$ & $-0.03(0.02)$ & 0.039 & 0.42 & 0.41 \\
\hline
\end{tabular}

Bonferroni corrected P-values

$* \mathrm{P}<0.05$

$* * P<0.01$

$* * * \mathrm{P}<0.001$

All models are adjusted for sex, age, sleep stage 2 duration, derivation, and time since sleep onset. Sleep spindle amplitude is referring to a standardized value within subjects across the night.

$B$ refers to unstandardized estimate

SE refers to Standard Error 


\section{Figure Legends}

Figure 1. Sleep spindle amplitudes in slow (black line) and fast (grey line) frequency ranges as a function of time since sleep onset in frontal (A) and central (B) derivations. X-axis refers to individually defined $z$-score decile for the time since sleep onset, and $Y$-axis refers to individually defined $z$-score of the sleep spindle amplitude across the night.

Figure 2. Sleep spindle amplitudes in the frequency range $9-16 \mathrm{~Hz}$ as a function of time since sleep onset and PGS for morningness divided in three groups based on 1 Standard Deviation $(\leq-1$ (dotted), $>-1$ and $<1$ (grey), $\geq+1$ (black). X-axis refers to individually defined $z$-score decile for the time since sleep onset, and $Y$ axis refers to individually defined $z$-score of the sleep spindle amplitude across the night.

Figure 3. Sleep spindle amplitudes in the frequency range $9-12.5 \mathrm{~Hz}$ as a function of time since sleep onset and PGS for morningness divided in three groups based on 1 Standard Deviation ( $\leq-1$ (dotted), $>-1$ and $<1$ (grey), $\geq+1$ (black). $X$-axis refers to individually defined $z$-score decile for the time since sleep onset, and $Y$ axis refers to individually defined $z$-score of the sleep spindle amplitude across the night. Panel $A$ : frontal derivation, panel $\mathrm{B}$ : central derivation. 
Spindles in frontal derivation

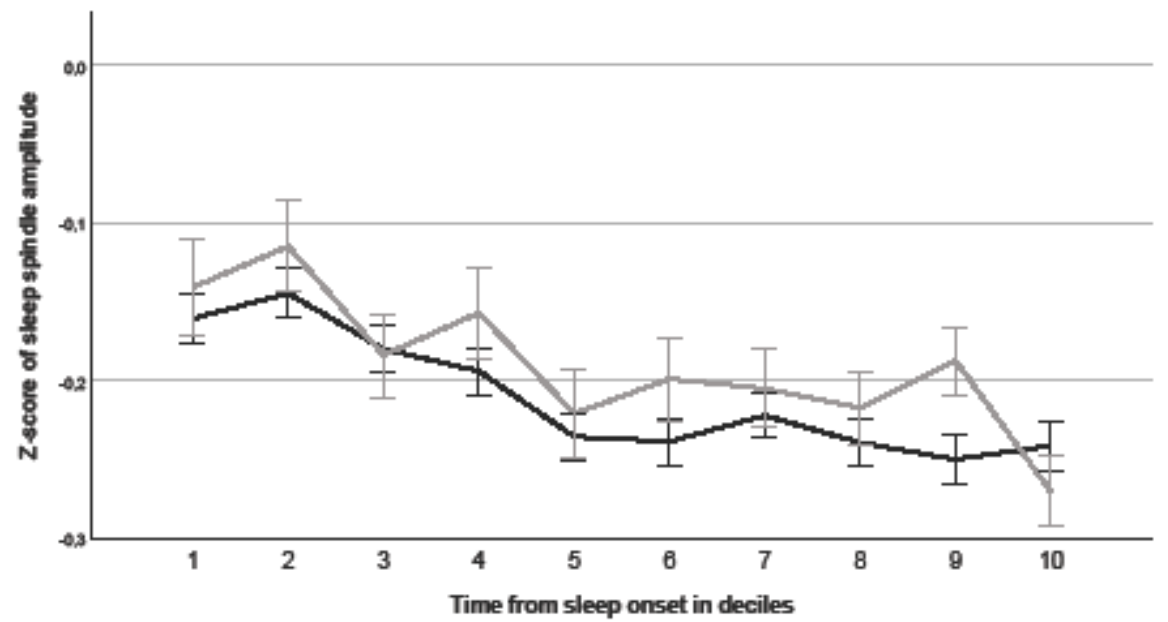

Page 1

This article is protected by copyright. All rights reserved. 


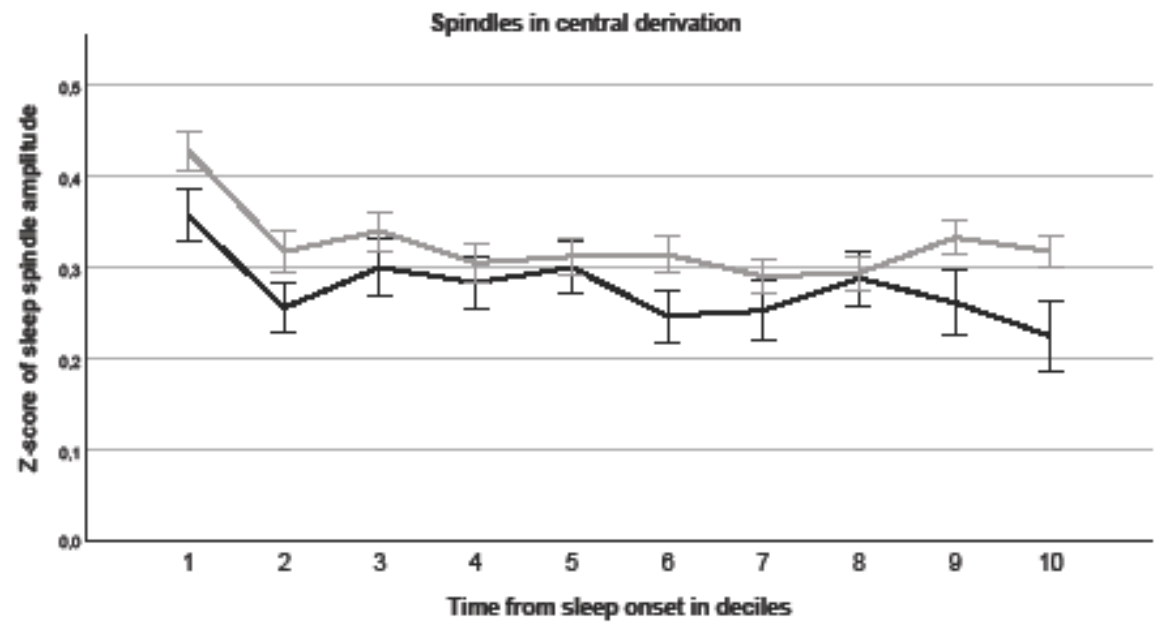

Page 1

This article is protected by copyright. All rights reserved. 


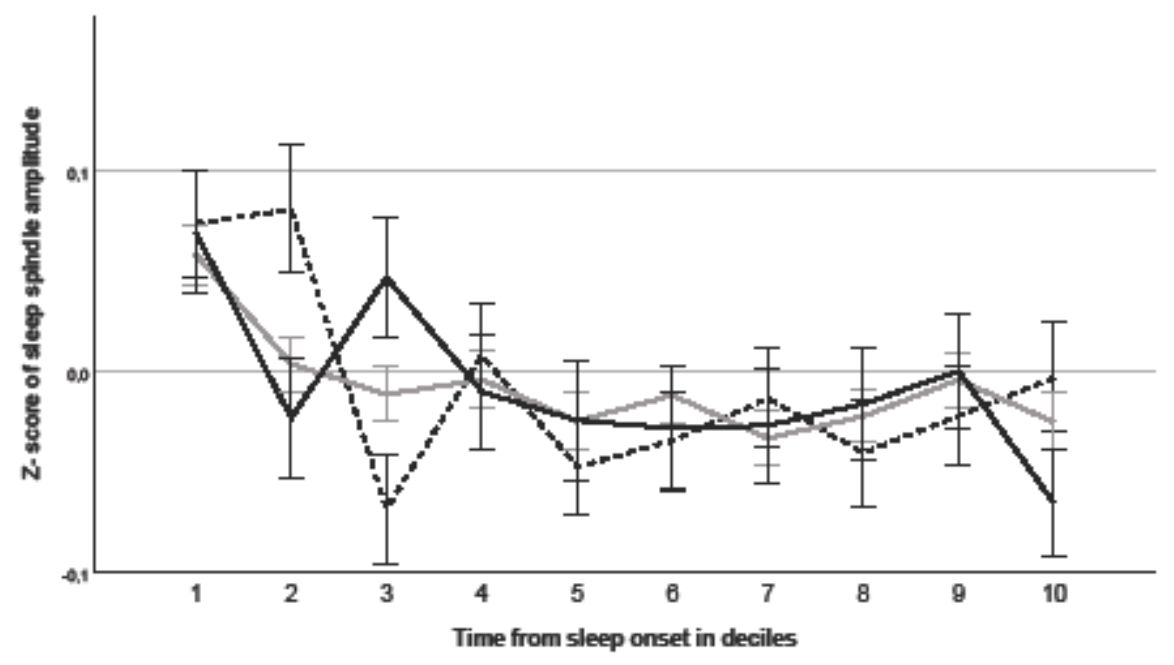

Page 1 
Slow spindles in frontal derivation

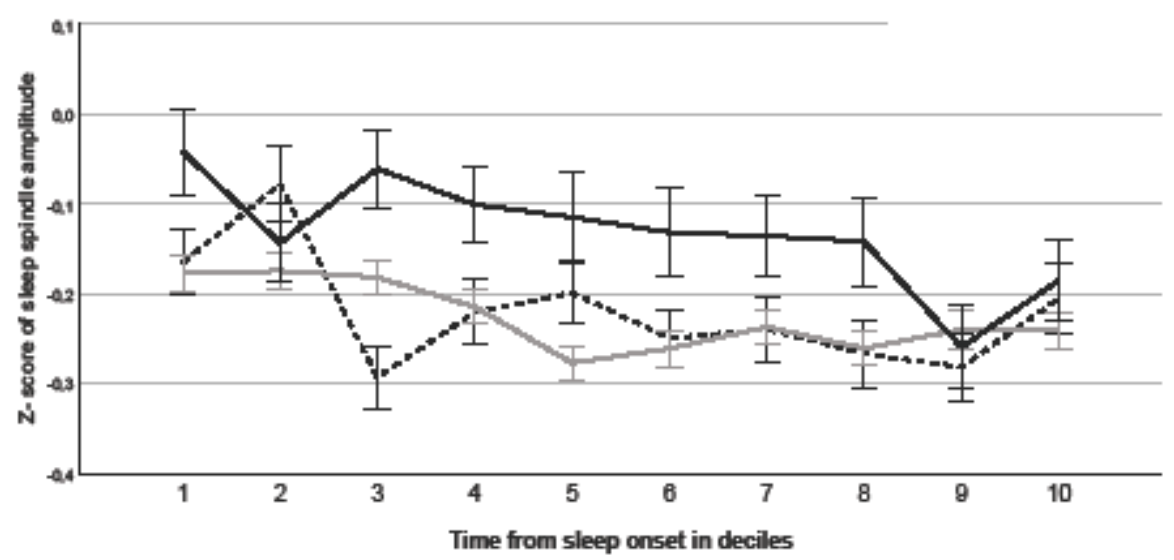

Page 1

This article is protected by copyright. All rights reserved. 
Slow spindles in central derivation

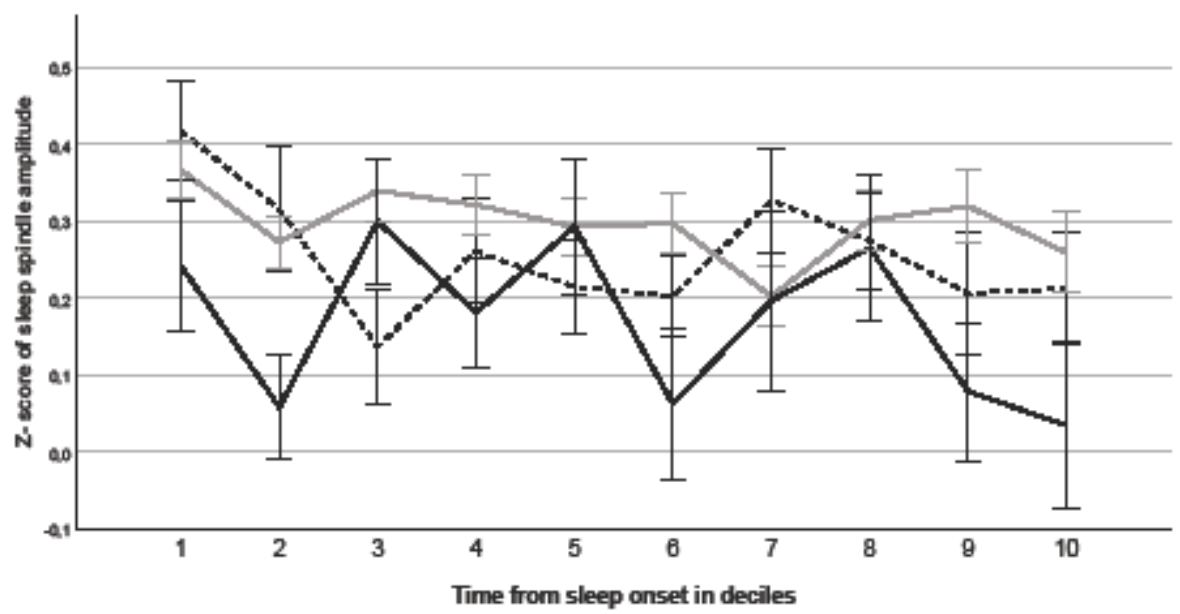

Page 1 